\title{
Correction to: Ductile Mode Cutting of Brittle Materials
}

\section{Correction to:}

K. Liu et al., Ductile Mode Cutting of Brittle Materials, Springer Series in Advanced Manufacturing, https://doi.org/10.1007/978-981-32-9836-1

In the original version of the book, the book author name was wrongly spelled in the chapter XML. The name should be KUI LIU. The erratum chapter has been updated with the change. 